\title{
Introduction du dossier « Autour du nouveau-né en maternité »
}

\author{
C. Francoual \\ Reçu le 22 avril 2009 ; accepté le 2 mai 2009 \\ (C) Springer-Verlag 2009
}

Bravo et continuez à vous battre ! Voici ce que j'ai eu envie de dire aux auteurs de ce dossier consacré à la pédiatrie en maternité. Pierre Lequien, quand il m'a fait l'amitié de me demander cet éditorial, savait bien qu'il toucherait chez moi un point sensible. En effet, il y a 40 ans, au moment où les réanimations néonatales se sont créées pour traiter les nouveau-nés atteints d'infections néonatales graves ou d'asphyxie périnatale sévère, il est apparu à certains d'entre nous, anciens réanimateurs, qu'il serait plus judicieux de se déplacer pour traiter ces nouveau-nés dès la salle de naissance, dans les maternités, et ainsi assurer leur sécurité. La deuxième bataille a été d'essayer de limiter les séparations mère-enfant par l'amélioration de la prise en charge des enfants en suite de couches et par la création d'unités néonatales mère-bébé (unités kangourou) à l'intérieur des maternités. Ensuite, la pédiatrie de maternité s'est vue attribuer d'autres missions, toutes importantes, comme la promotion de l'allaitement maternel, le dépistage des difficultés de l'attachement mère-enfant et de la dépression du post-partum, l'établissement de liens avec la PMI et les services sociaux, la promotion de la santé. Les deux articles de cette revue consacrés à l'allaitement maternel
(G. Gremmo-Féger) et aux soins au nouveau-né normal (B. Branger), montrent bien la difficulté inhérente à ce travail : toujours trouver le juste milieu entre trop de médicalisation qui va à l'encontre de l'adaptation naturelle du nouveau-né à son nouveau milieu et un courant de démédicalisation qui pourrait nuire à la santé de l'enfant. L'article sur le travail du psychologue (Reine Van der Linden) met l'accent sur l'importance de la qualité des liaisons entre les différents professionnels de la naissance, facteur indispensable de cohérence pour les familles. Le travail de pédiatre de maternité, malgré son importance reconnue de façon unanime, est pourtant comme l'indique si bien le Pr JP Dubos, le premier menacé en cas de « crise » au profit d'activités plus directement rentables. Les objectifs de la prise en charge des bébés et de leurs mères sont maintenant bien établis : médicalisation appropriée, limitation de la séparation mèreenfant, promotion de l'allaitement maternel et dépistage des difficultés de l'attachement. Leur mise en œuvre reste fragile et mérite une attention constamment renouvelée. Les plus jeunes devront se battre pour les défendre avec enthousiasme et imagination même si la place du pédiatre de maternité est destinée à connaître des changements.

Note de l'éditeur : En raison des délais, le dossier «Autour du nouveau-né en maternité » a dû faire l'objet d'une publication en deux parties dans les numéros de juin (Vol. $1 \mathrm{~N}^{\circ}$ 2) et de septembre (Vol. $1 \mathrm{~N}^{\circ}$ ) de la Revue de médecine périnatale. Nous vous remercions de votre compréhension.

C. Francoual $(\bowtie)$

Maternité Saint-Vincent-de-Paul, 82, avenue Denfert-Rochereau,

F-75014 Paris, France

e-mail : christine.francoual@svp.aphp.fr 